\title{
Ruptura simultânea do tendão quadricipital e do tendão patelar contralateral em uma paciente com insuficiência renal crônica
}

\author{
Simultaneous rupture of the quadriceps tendon and contralateral patellar tendon in a patient \\ with chronic renal failure
}

\begin{abstract}
Alfredo dos Santos Netto ${ }^{1}$, Leandro Jun Aihara ${ }^{2}$, Yuri Yamada ${ }^{3}$, Pedro Sire Salgado ${ }^{3}$, Osmar Pedro Arbix de Camargo ${ }^{4}$, Ricardo de Paula Leite Cury ${ }^{5}$
\end{abstract}

\section{Resumo}

A ruptura simultânea do tendão quadricipital e do tendão patelar contralateral representa uma associação extremamente rara, e poucos casos foram reportados na literatura. Doenças sistêmicas predispõe a ruptura tendinosa espontânea, como insuficiência renal crônica, hiperparatireoidismo, artrite reumatoide, gota e lúpus eritematoso sistêmico. Nós reportamos o caso de uma mulher de 40 anos, com insuficiência renal crônica dialítica e hiperparatireoidismo secundário, que sofreu uma ruptura espontânea e simultânea do tendão patelar de um joelho e do tendão quadricipital do outro. O reparo da lesão dos tendões patelar e quadricipital foi realizado através de sutura não absorvível com pontos transósseos, e o reparo do tendão patelar foi complementado com a confecção de um dispositivo de proteção dinâmica. Boa recuperação funcional foi obtida em ambos os joelhos.

1. Professor Instrutor da Faculdade de Ciências Médicas da Santa Casa de São Paulo - Departamento de Ortopedia e Traumatologia 2. Médico Assistente da Irmandade da Santa Casa de Misericórdia de São Paulo - Departamento de Ortopedia e Traumatologia

3. Acadêmico da Faculdade de Ciências Médicas da Santa Casa de São Paulo $-4^{\circ}$ Ano do Curso de Graduação em Medicina

4. Professor Adjunto da Faculdade de Ciências Médicas da Santa Casa de São Paulo - Departamento de Ortopedia e Traumatologia - Grupo de Cirurgia do Joelho

5. Professor Instrutor da Faculdade de Ciências Médicas da Santa Casa de São Paulo - Departamento de Ortopedia e Traumatologia. Chefe do Grupo de Cirurgia do Joelho

Trabalho realizado: Faculdade de Ciências Médicas da Santa Casa de São Paulo. Departamento de Ortopedia e Traumatologia / Irmandade da Santa Casa de Misericórdia de São Paulo. Departamento de Ortopedia e Traumatologia

Endereço para correspondência: Alfredo dos Santos Netto. Rua Doutor Cesário Mota Júnior, 112. Vila Buarque - 01221-020 São Paulo - SP - Brasil. Tel /Fax: (11) 3064-1520 ou 3064-2035. E-mail:alfredo.santos@fcmsantacasasp.edu.br

Conflito de interesses: Todos os autores declaram não haver nenhum potencial conflito de interesses referente a este artigo.
Este caso enfatiza a importância do hiperparatireoidismo secundário na etiologia das rupturas tendinosas, em pacientes com insuficiência renal crônica.

Descritores: Joelho, Ligamento patelar, Insuficiência renal, Ruptura

\begin{abstract}
"Simultaneous quadriceps and patellar tendon rupture is rare, and few cases were reported in the literature. Spontaneous tendon ruptures tend to increase considerably in patients with metabolic diseases such as chronic renal failure, hyperparathyroidism, rheumatoid arthritis, gout, and systemic lupus erythematosus. The reported case regards a 40-year-old woman with spontaneous and simultaneous quadriceps and contra-lateral patellar tendon rupture. The patient suffered from chronic renal failure and hyperparathyroidism. She was treated surgically by osseotendinous repair with nonabsorbable suture, the patelar tendon repair was complemented with a dynamic protection device. Good functional recovery was achieved on both knees. This case emphasizes the high parathyroid hormone level in the etiology of tendons ruptures, in patients with chronic renal failure."
\end{abstract}

Keywords: Knee, Patellar ligament, Renal insufficiency, Rupture

\section{Introdução}

A ruptura do tendão patelar geralmente ocorre em pacientes com menos de 40 anos, que realizam atividades esportivas, e fatores como tendinite patelar prévia e injeções locais de corticoesteróides aumentam o risco desta lesão ${ }^{(1-4)}$. A ruptura do tendão quadricipital ocorre com maior frequência em pacientes na sexta ou sétima décadas de vida, com alterações degenerativas no tendão, após trauma ou queda $\mathrm{a}^{(1-4)}$. 
Aruptura simultânea do tendão quadricipital e do tendão patelar contralateral representa uma associação extremamente rara, e poucos casos foram reportados na literatura ${ }^{(1-5)}$. Neste artigo, reportamos o caso de uma mulher de 40 anos, com insuficiência renal crônica dialítica, que sofreu uma ruptura espontânea e simultânea do tendão patelar de um joelho e do tendão quadricipital do outro.

\section{Descrição do Caso}

Uma mulher de 40 anos de idade deu entrada em nosso pronto socorro com quadro de dor e impotência funcional em ambos os joelhos, sem história de trauma. Ao exame físico de entrada, apresentava aumento de volume e incapacidade para extensão ativa de ambos os joelhos, com "gaps" à palpação do tendão patelar direito, e do tendão quadricipital esquerdo Fig. 1.

A paciente tinha antecedente de insuficiência renal crônica, e vinha realizando hemodiálise há 5 anos. Exames laboratoriais realizados dois meses antes da lesão demonstravam níveis séricos de paratormônio de $1.094 \mathrm{pg} / \mathrm{ml}$ (normal: $10-65 \mathrm{pg} / \mathrm{ml}$ ), sugerindo a presença de hiperparatireoidismo secundário ${ }^{(6)}$.

O exame radiográfico dos joelhos revelou ausência de fraturas, com patela alta no lado direito e baixa à esquerda. O exame de ressonância nuclear magnética evidenciou lesão do tendão patelar direito, tipo desinserção no pólo inferior da patela, e lesão do tendão quadricipital esquerdo, também do tipo desinserção Fig 2.

A paciente foi internada e submetida a tratamento cirúrgico cinco dias após a lesão. As duas lesões foram abordadas no mesmo ato operatório. Após incisão anterior e exposição das lesões, observou-se uma

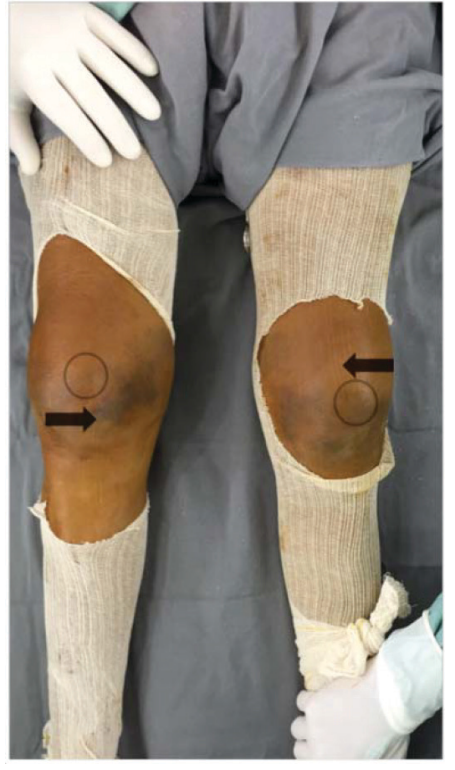

Figura 1 - Imagem clínica pré-operatória. A posição das patelas está destacada pelos círculos pretos. Nota-se ascensão significativa da patela direita em relação à esquerda. As setas indicam o local dos "gaps" palpáveis em ambos os tendões.

lesão do tipo desinserção dos tendões patelar direito e quadricipital esquerdo, sem degeneração tendinosa evidente Fig 3. Foi realizada sutura transóssea dos tendões com fio não absorvível pela técnica de Krackow et al, $1986^{(7)}$. Foram confeccionados 3 túneis verticais na patela, e os fios de sutura foram passados pelos túneis ósseos, sendo amarrados no lado oposto da patela. $\mathrm{O}$ retináculo também foi reparado com sutura contínua não absorvível. No lado direito, após a reparação das estruturas rompidas, realizamos a confecção de um dispositivo de proteção, conforme preconizado por Frutos et al(2009)(8) Fig 4. As radiografias realizadas
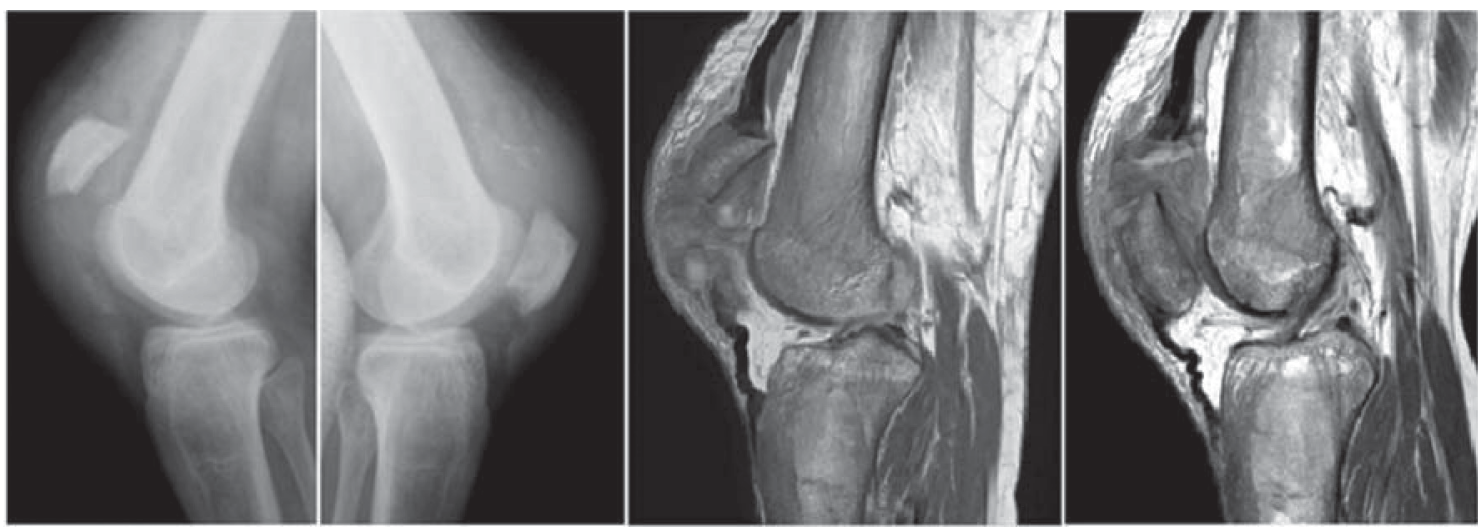

Figura 2 - À esquerda, radiografias evidenciando patela alta no joelho direito, com índice de Caton-Deschamps de 2.0, e patela baixa no joelho esquerdo, com índice de Caton-Deschamps de 0.6. Ao centro, corte sagital de ressonância magnética do joelho direito que evidencia desinserção do tendão patelar no ápice da patela com ascensão da mesma, associada a infiltrado hemorrágico em torno da lesão. À direita, corte sagital de ressonância magnética do joelho esquerdo que mostra desinserção do tendão quadricipital na base da patela, com afrouxamento do tendão patelar e abaixamento da patela, associado a infiltrado hemorrágico em torno da lesão. 

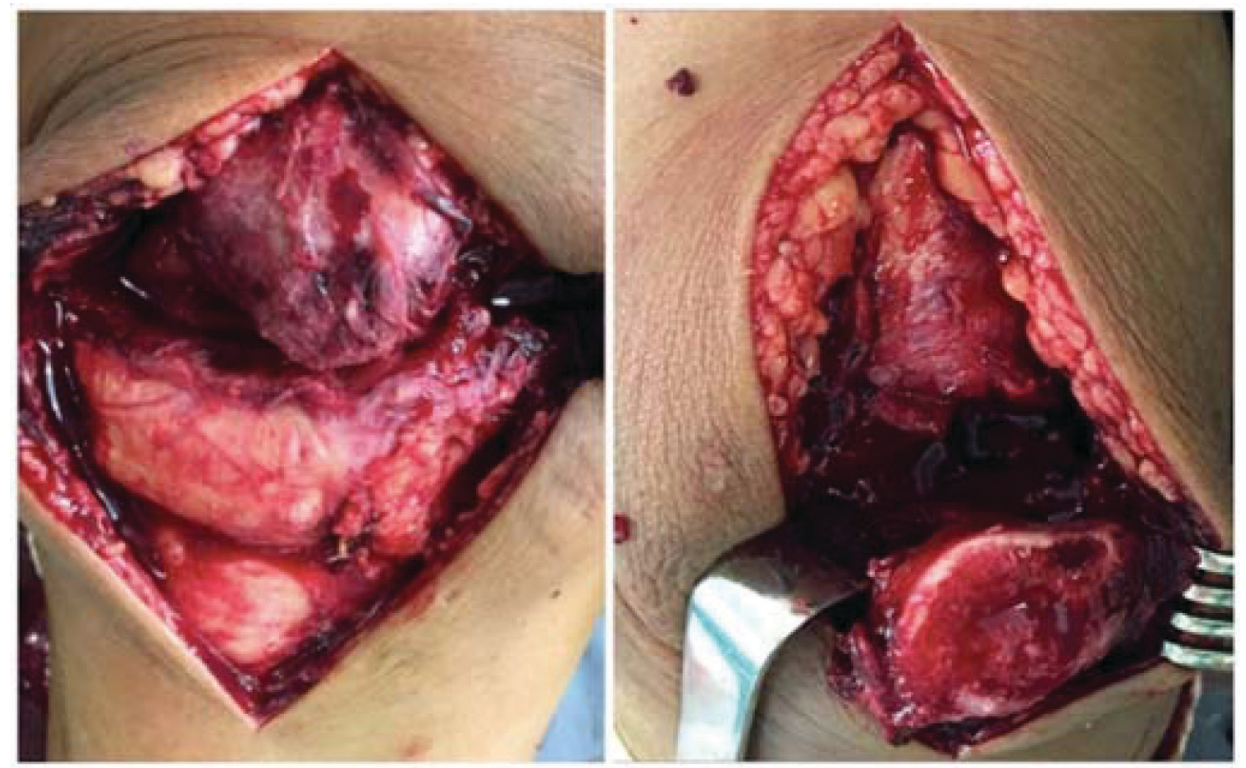

Figura 3 - Imagens do intra-operatório mostrando na foto à esquerda, desinserção do tendão patelar do joelho direito, e na foto à direita, desinserção do tendão quadricipital do joelho esquerdo, sem degeneração evidente dos tendões.
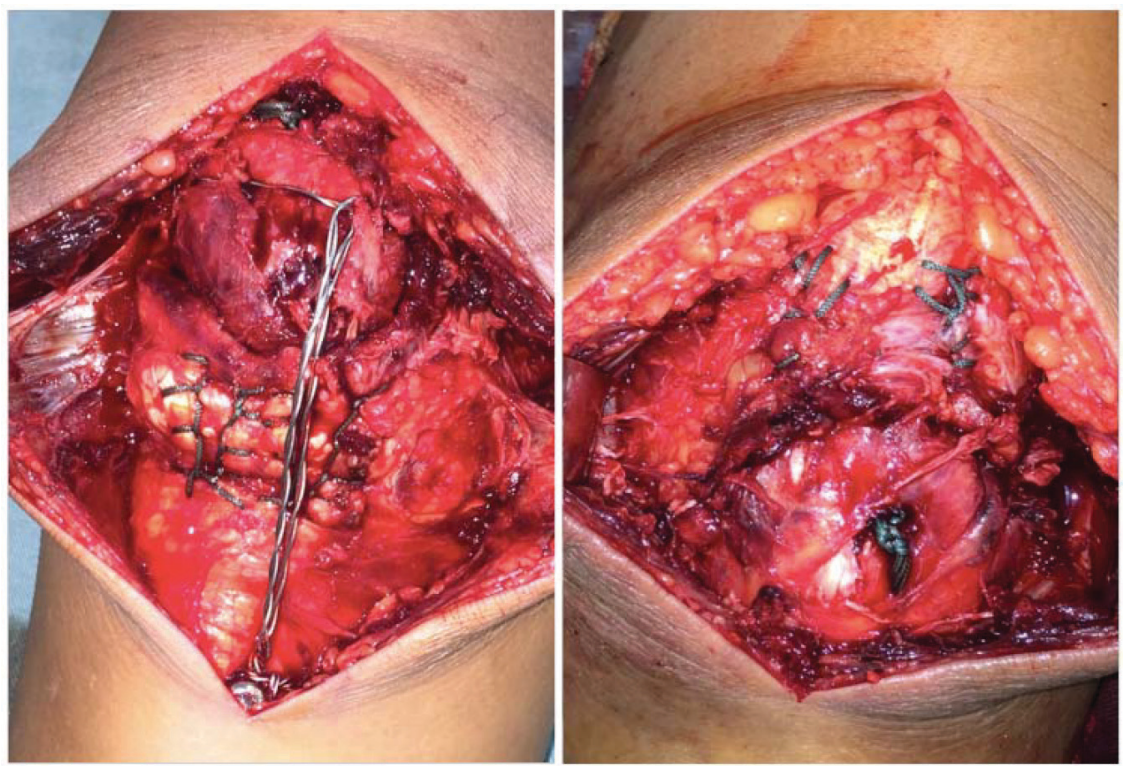

Figura 4 - Imagens após ancoragem das suturas e confecção do dispositivo de proteção do reparo do tendão patelar direito, na foto à esquerda, e após sutura com pontos transósseos do tendão quadricipital esquerdo, na foto à direita.

no pós-operatório evidenciaram restabelecimento da altura patelar bilateral Fig 5 .

No pós-operatório, ambos os joelhos foram imobilizados com "brace" por 60 dias. Sete dias após a cirurgia, foram iniciados exercícios isométricos para quadríceps. Mobilização passiva foi iniciada precocemente no lado direito, pela presença do mecanismo de proteção dinâmica, e após 1 mês no lado esquerdo. Após 6 semanas a paciente iniciou carga parcial com muletas e mobilização ativa. Com 4 meses a paciente conseguia andar com carga total e auxílio de 1 muleta.

\section{Discussão}

Várias doenças sistêmicas predispõem a ruptura tendinosa espontânea, como insuficiência renal crônica, hiperparatireoidismo, artrite reumatoide, gota e lúpus eritematoso sistêmico ${ }^{(1,3-5,9-10)}$.

A ruptura simultânea do tendão quadricipital e do tendão patelar contralateral é uma lesão rara ${ }^{(1-5)}$. Encontramos relatos na literatura dessa lesão em pessoas saudáveis, sem doenças sistêmicas ${ }^{(2,4)}$, e encontramos casos, como o nosso, em que a lesão ocorreu 


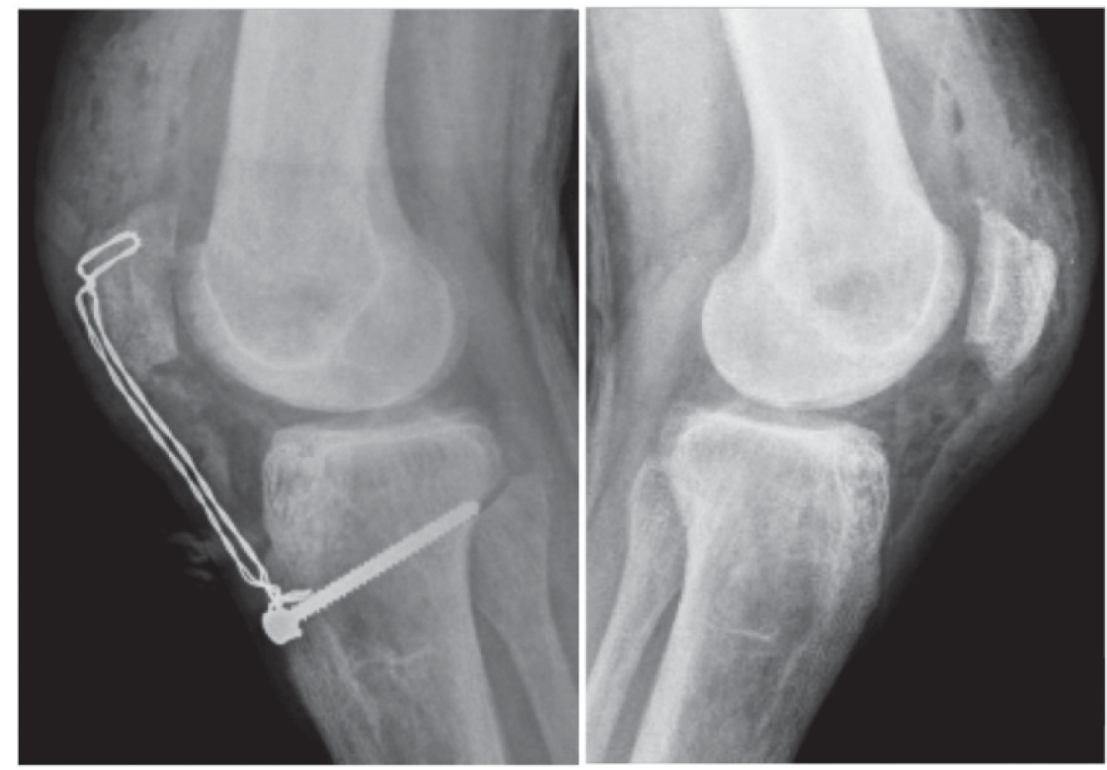

Figura 5 - Radiografias realizadas no pós-operatório demonstrando restabelecimento da altura patelar. (índice de Caton Deschamps de 0.9 do lado direito e de 1.0 do lado esquerdo)

em pacientes com doença renal crônica dialítica ${ }^{(1,3,5)}$.

Existem várias teorias a respeito da patogênese da ruptura de tendões em pacientes que realizam hemodiálise. Falência renal, com necessidade de hemodiálise, pode causar várias complicações, como amiloidose e osteodistrofia renal ${ }^{(1,3,6)} \cdot \mathrm{Na}$ amiloidose associada com a hemodiálise, existe uma produção anormal de beta-2 microglobulina, que se deposita em algumas áreas, como as articulações, ossos e tendões. O depósito desta substância leva a redução da elasticidade dos tendões e predispõe a rupturas após mínimo estresse ${ }^{(1,3)}$. Apesar de não termos realizado uma análise histoquímica do tecido tendinoso de nossa paciente, é improvável que essa tenha sido a causa de suas lesões. A amiloidose associada com a hemodiálise costuma se apresentar com outras manifestações musculoesqueléticas, como a síndrome do túnel do carpo, dor no ombro e tenossinovite flexora nas mãos, achados não encontrados em nossa paciente $^{(1,3)}$. A osteodistrofia renal com hiperparatireoidismo secundário cursa com osteoporose generalizada, reabsorção óssea subperiosteal e fraqueza da junção osteotendínea ${ }^{(1,3,5-6,9-10)}$. Essa condição tem sido apontada como a principal causa de ruptura tendinosa em pacientes com doença renal, e é muito provável que tenha sido a causa da lesão de nossa paciente. Um exame realizado dois meses antes de sua internação apontava nível sérico de paratormônio elevado, compatível com hiperparatireoidismo secundário à insuficiência renal crônica. Os achados intra-operatórios também foram sugestivos desta condição. A presença de ruptura na junção osteotendínea sugere que a causa das lesões tenha sido o hiperparatireoidismo crônico e mal controlado, levando a fragilidade da junção osteotendínea. Rupturas associadas com degeneração ou patologia tendinosa geralmente ocorrem no tendão ou na junção miotendínea ${ }^{(1,3)}$.

O diagnóstico e tratamento cirúrgico precoce das lesões do mecanismo extensor são importantes para um resultado clínico favorável ${ }^{(3,5)}$. O reparo das lesões dos tendões patelar e quadricipital, através de sutura não absorvível com pontos transósseos, foi o método realizado neste caso, e é o método mais utilizado na literatura ${ }^{(3)}$. Nas lesões da porção distal do mecanismo extensor, entre o ápice da patela e a tuberosidade tibial, a reparação é sujeita a forças de tração muscular intensas, que podem levar à diástase das estruturas reparadas. A utilização de dispositivos que protegem a reparação é uma opção nesse tipo de lesão. Em nosso caso realizamos a confecção de um dispositivo de proteção, conforme preconizado por Frutos et al, $2009^{\left({ }^{(8)}\right.}$.

\section{Comentários Finais}

A avaliação clínica cuidadosa de pacientes com doença renal crônica e queixa de dor nos joelhos é essencial, permitindo diagnóstico e tratamento cirúrgico precoce das lesões. O controle clínico adequado desses pacientes é fundamental para prevenção de lesões como a de nossa paciente ${ }^{(5)}$.

\section{Referências}

1. Grecomoro G, Camarda L, Martorana U. Simultaneous chronic rupture of quadriceps tendon and contra-lateral patellar tendon in a patient affected by tertiary hyperparatiroidism. J Orthop Traumatol. 2008; 9(3):159-62. 
2. Rogers A, Rix S, Kulkarni R. Simultaneous rupture of a patellar tendon and contralateral quadriceps tendon in a healthy individual. Orthopedics. 2003; 26(8):817-8.

3. Muratli HH, Celebi L, Hapa O, Biçimoğlu A. Simultaneous rupture of the quadriceps tendon and contralateral patellar tendon in a patient with chronic renal failure. J Orthop Sci. 2005; 10(2):227-32.

4. Munshi NI, Mbubaegbu CE. Simultaneous rupture of the quadriceps tendon with contralateral rupture of the patellar tendon in an otherwise healthy athlete. Br J Sports Med. 1996; 30(2):177-8

5. Loehr J, Welsh RP. Spontaneous rupture of the quadriceps tendon and patellar ligament during treatment for chronic renal failure. Can Med Assoc J. 1983;129(3):254-6.

6. Martins CTB, Jorgetti V. Hiperparatireoidismo secundário e insuficiência renal crônica: impacto na resposta imune. J Bras Nefrol. 1995; 17(2):85-9.
7. Krackow KA, Thomas SC, Jones LC. A new stitch for ligamenttendon fixation. Brief note. J Bone Joint Surg Am. 1986;68(5):7646.

8. Frutos CFA, Camargo OPA, Severino NR, Cury RPL, Oliveira VM, Aihara T, et al. Dispositivo de proteção após reparação das rupturas do mecanismo extensor do joelho. Rev Bras Ortop. 2009; 44(1):57-60.

9. Gao MF, Yang HL, Shi WD. Simultaneous bilateral quadriceps tendon rupture in a patient with hyperparathyroidism undergoing long-term haemodialysis: a case report and literature review. J Int Med Res. 2013; 41(4):1378-83.

10. Chen CM, Chu P, Huang GS, Wang SJ, Wu SS. Spontaneous rupture of the patellar and contralateral quadriceps tendons associated with secondary hyperparathyroidism in a patient receiving long-term dialysis. J Formos Med Assoc. 2006; 105(11):941-5

Trabalho recebido: 18/04/2017

Trabalho aprovado: 26/09/2017 